\title{
Magnetoestratigrafía de la Formación San Marcos del Cretácico Inferior, Coahuila, México: Implicaciones tectónicas y paleogeográficas
}

\author{
Irving Rafael Arvizu-Gutiérrez ${ }^{1}$, Gildardo Alonso González-Naranjo", \\ Roberto Stanley Molina-Garza ${ }^{2,}$, Gabriel Chávez-Cabello ${ }^{3}$ \\ ${ }^{1}$ Posgrado en Ciencias de la Tierra, Centro de Geociencias, Universidad Nacional Autónoma de México, Campus Juriquilla, Apartado \\ postal 1-742, Querétaro, Querétaro, 76001, México. \\ ${ }^{2}$ Centro de Geociencias, Universidad Nacional Autónoma de México, Campus Juriquilla, \\ Apartado postal 1-742, Querétaro, Querétaro, 76001, México \\ ${ }^{3}$ Facultad de Ciencias de la Tierra, Universidad Autónoma de Nuevo León, Linares. \\ *rmolina@geociencias.unam.mx
}

\begin{abstract}
Resumen
Se realizó un estudio magnetoestratigráfico de la Formación San Marcos en la parte central de Coahuila, en dos localidades a lo largo de la Falla San Marcos. En estas localidades aflora una secuencia de conglomerados, areniscas inmaduras y lodolitas rojas, cementadas por hematita; esta secuencia de ambiente continental está asignada al Cretácico Temprano. Todos los especímenes, de un total de 35 sitios, fueron sujetos a procesos de desmagnetización por campos alternos y térmico, siendo este último el más efectivo para aislar la magnetización característica (alta temperatura). La componente característica fue aislada entre $625^{\circ} \mathrm{y} 660^{\circ} \mathrm{C}$, se identificaron magnetizaciones de polaridades normal e inversa con una dirección media general de $D e c=352.7^{\circ}$, Inc $=55.5^{\circ}, N=3$ localidades (18 sitios), $k=27.07, \alpha_{95}=6.8^{\circ}$. Este resultado sugiere una pequeña rotación horaria con respecto la dirección promedio del Cretácico esperada, calculada de la curva de vagabundeo polar aparente para Norteamérica. Sin embargo, los datos sugieren que existieron rotaciones relativas entre las localidades muestreadas y que las rotaciones son respecto a ejes verticales locales. La zonación de polaridad magnética identificada sugiere que la edad aproximada de la Formación San Marcos comprende desde el Barremiano Temprano al Aptiano Temprano. La correlación con la escala magnética de polaridad global sugiere que esta unidad se relaciona con la Formación Cupido y que el depósito de las facies transgresivas litorales de la Formación Las Uvas y la inundación de la Paleo-isla de Coahuila en la localidad de Potrero Colorado se produjeron durante el Aptiano Temprano. La mejor correlación con la escala magnética global de polaridad sugiere que la zonación observada corresponde al intervalo de M5n (normal) a M0r (inverso) dentro de un rango de aproximadamente 131-125 Ma. La tasa de sedimentación que indica esta correlación, asumiendo una tasa constante, es relativamente baja $(\sim 30-50 \mathrm{~m} / \mathrm{Ma})$ para un ambiente de tectónica activa y es más acorde a regiones donde el acomodamiento se debe a ajustes isostáticos entre bloques corticales (Bloque de Coahuila y Cuenca de Sabinas).
\end{abstract}

Palabras clave: Cretácico, Coahuila, Cuenca de Sabinas, magnetoestratigrafía.

\begin{abstract}
We report the results of a magnetostratigraphic study of the San Marcos Formation in central Coahuila, at two localities along the San Marcos Fault. These localities are characterized by outcrops of a sequence of conglomerates, immature sandstone and red mudstone, cemented by hematite; the sequence was deposited in a continental environment, and has been assigned to the Early Cretaceous. All specimens, from a total of 35 paleomagnetic sites, were subjected to alternating field and thermal demagnetization, the latter being the most effective in isolating the (high temperature) characteristic magnetization. The characteristic component is unblocked between $625^{\circ}$ and $660^{\circ} \mathrm{C}$. Both normal and reverse polarity magnetizations are represented, and the overall mean is of Dec $=352.7^{\circ}$, Inc $=55.5^{\circ}$,
\end{abstract}


$N=3$ localities (18 sites), $k=27.07, \alpha_{95}=6.8^{\circ}$. This result suggests a small clockwise rotation with respect to the expected reference direction for the Cretaceous, estimated from the apparent polar wandering curve for cratonic North America. However, the data suggest that there was relative rotation between localities, and the observed rotations are more likely explained as local vertical-axis rotations. The magnetic polarity zonation in the San Marcos Formation suggests that this unit represents the Early Barremian through Early Aptian. The correlation to global magnetic polarity timescale suggests that this unit is correlative with Cupido Formation, and that the transgressive littoral facies of the Las Uvas Formation, as well as flooding of Coahuila paleo-island at Potrero Colorado, occurred in the Early Aptian. The best correlation with the global magnetic polarity time scale corresponds to the interval from M5n (normal) to MOr (inverse) approximately between 131-125 Ma. The sedimentation rate indicated by this correlation, assuming a constant rate, is relatively low ( 30-50 $\mathrm{m} / \mathrm{Ma}$ ) for an active tectonic environment and is more consistent with a region where accommodation is achieved by isostatic adjustment between crustal blocks (Coahuila Block and Sabinas Basin).

Keywords: Cretaceous, Coahuila, Sabinas Basin, magnetostratigraphy.

\section{Introducción}

La sedimentación durante el Mesozoico en el noreste de México está fuertemente controlada por una configuración del basamento heredada de una tectónica extensional ligada a la apertura del Golfo de México (Wilson, 1990; Goldhammer, 1999; Eguiluz de Antuñano, 2001). Este artículo provee información adicional sobre la evolución de los sistemas deposicionales de esta región en el Cretácico Temprano. Nuestro conocimiento de la evolución paleogeográfica del NE de México está basado, principalmente, en el estudio de secuencias marinas clásticas y carbonatadas (Salvador, 1987; Goldhammer, 1999; Lehmann et al., 1999), ya que su contenido faunístico permite establecer edades relativas con cierta precisión. Las secuencias continentales depositadas en las márgenes de los bloques emergidos han recibido, en cambio, menos atención posiblemente debido a dificultades en determinar su edad de forma precisa. Los registros continentales pueden proporcionar información adicional sobre condiciones tectónicas regionales, por la respuesta combinada de los sistemas fluviales a cambios en el nivel base y en el relieve en las zonas emergidas. Tal es el caso de la Formación San Marcos, en el sur-centro de Coahuila, depositada en la margen norte de la Paleo-isla de Coahuila (Figura 1).

La Formación San Marcos consiste principalmente de arenisca inmadura granular, cementada por hematita. El nombre de Formación San Marcos fue propuesto por McKee et al. (1990) para la unidad que Imlay (1940) había llamado Arcosa San Marcos y que aflora en el valle San Marcos (VSM) al norte de la falla del mismo nombre. Aunque a la Formación San Marcos se le ha asignado una edad del Cretácico Temprano (Neocomiano), en base a correlaciones litoestratigráficas, su edad precisa no se conoce. Eguiluz de Antuñano (2001) sugiere que en el subsuelo de la Cuenca de Sabinas la Formación San Marcos cambia lateralmente hacia las facies de carbonatos de alta energía de la Formación Menchaca, cuya edad biostratigráfica corresponde al Berriasiano (Imlay, 1940). La Formación San Marcos sobreyace la Formación Barril Viejo en el subsuelo de la Cuenca de Sabinas y en el anticlinal de La Gavia, a unos
$75 \mathrm{~km}$ al sureste del valle de San Marcos. La Formación Barril Viejo contiene fauna del Hauteriviano (Eguiluz de Antuñano, 2001).

La Falla de San Marcos (FSM) en Coahuila central está íntimamente ligada al depósito de la Formación San Marcos, en el margen sur de la Cuenca de Sabinas. La falla fue definida primeramente por Charleston (1981) quién sugirió, al igual que Padilla y Sánchez (1982), desplazamientos laterales izquierdos durante el Jurásico a lo largo de la falla mediante interpretación de imágenes de satélite. Los primeros trabajos a detalle realizados dentro de la región de la FSM son de McKee et al. (1984, 1990), los cuales determinaron períodos de actividad de la falla en el Jurásico, el Cretácico Temprano y el Terciario, así como periodos de inactividad en el Jurásico más tardío y durante la mayor parte del Cretácico. El énfasis del trabajo de McKee y colaboradores es en la estratigrafía y proveniencia de la cuña clástica marina y continental del Mesozoico depositada al norte de la Falla de San Marcos, en el bloque hundido, producto de la misma actividad de la falla.

El objetivo de realizar un estudio de magnetoestratigrafia en la Formación San Marcos tiene tres propósitos principales: (1) tener una edad más precisa del depósito de la formación, (2) establecer con mejor precisión su correlación con depósitos marinos, y (3) conocer la tasa de sedimentación y así dilucidar las condiciones que dieron lugar al depósito. Actualmente se puede acotar la edad de la Formación San Marcos entre el Titoniano y el Aptiano, por el contenido de amonites (McKee et al. 1990) dentro de las Capas Tanque y Cuatro Palmas que la subyacen, y, por la Formación Cupido que le sobreyace. Según Lehmann et al. (1999), a través de estudios bioestratigráficos y estratigrafía isotópica, la Formación Cupido es una unidad transgresiva en un rango temporal del Hauteriviano al Aptiano (Cretácico Temprano). Al sobreyacer a la Formación San Marcos, existe un periodo de tiempo muy grande en el cual se puede ubicar estratigráficamente la Formación San Marcos dentro del Neocomiano (McKee et al., 1990). Conocer con mejor detalle las condiciones de depósito de la Formación San Marcos es importante por ser interpretada ésta como el registro de una etapa de reactivación de la FSM en el 


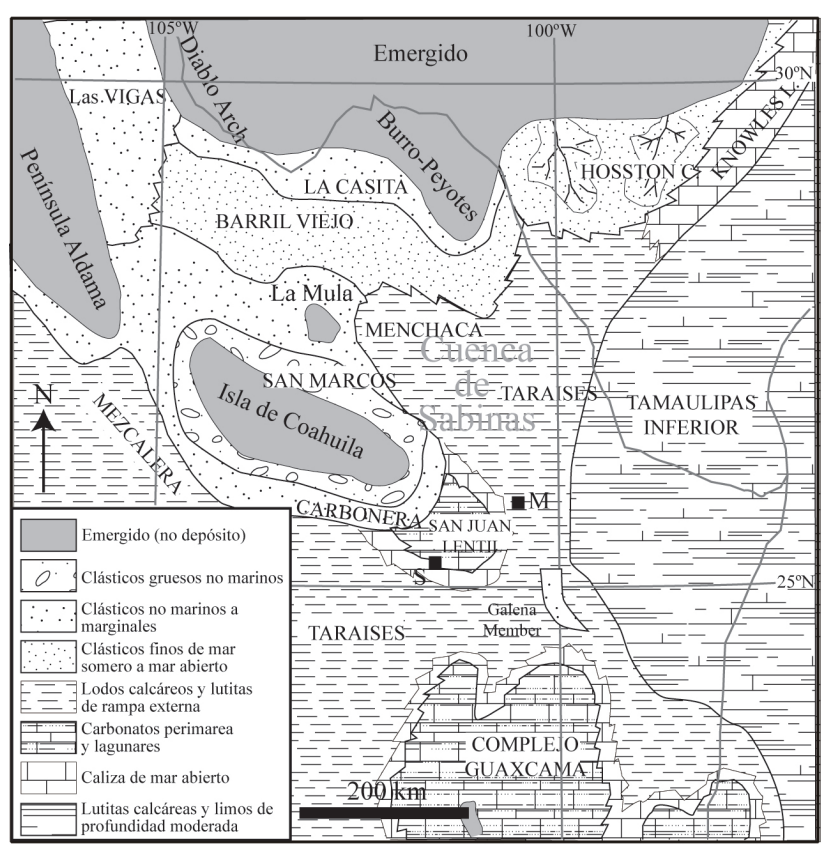

SW

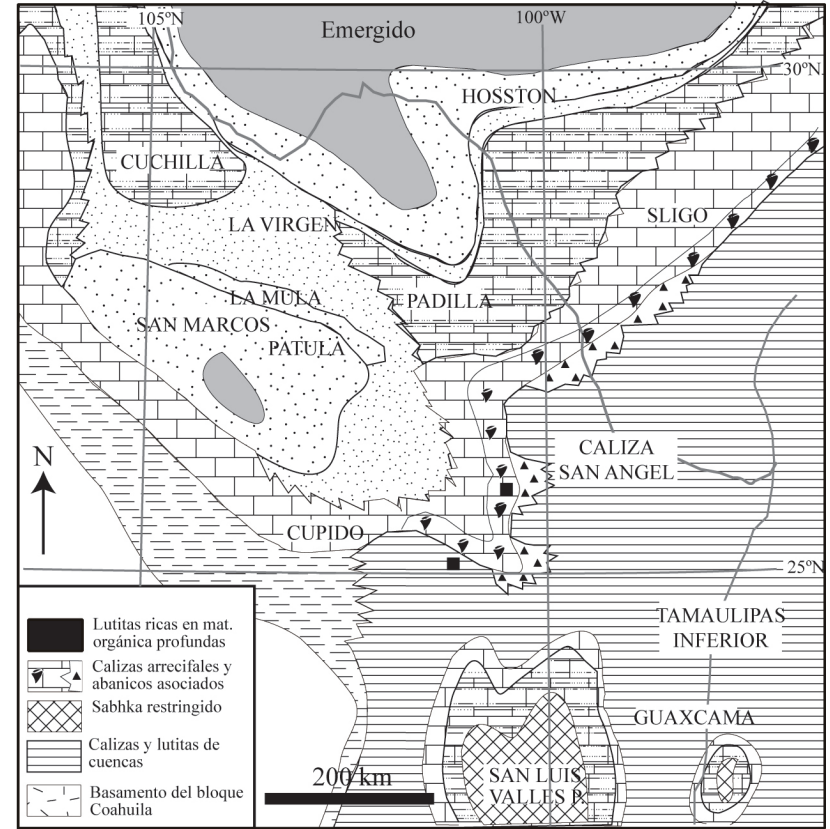

$\mathrm{NE}$

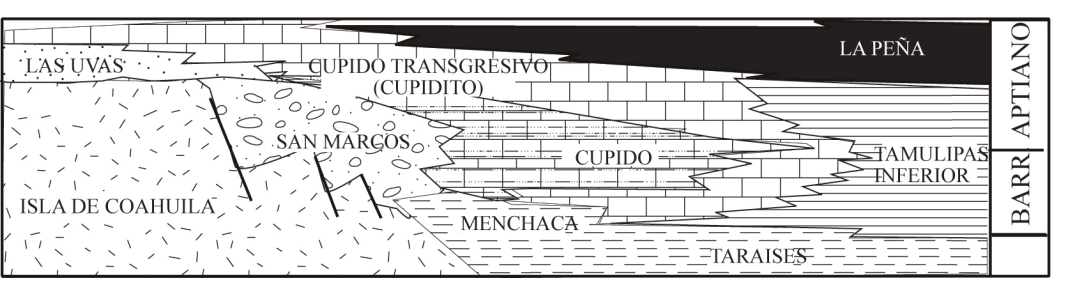

Figura 1. Paleogeografía del NE de México para el Cretácico Temprano (Hauteriviano a la izquierda y Barremiano a la derecha) y una sección cronoestratigráfica de la isla de Coahuila a la Cuenca de Sabinas. Modificado de Goldhammer (1999) y Lehmann et al. (1999).

Cretácico Temprano (McKee et al., 1990) y por ende de rejuvenecimiento de la Cuenca de Sabinas.

El uso de la magnetoestratigrafía como herramienta de correlación y fechamiento se basa en dos principios generales. El primero es que, en el momento de su formación, las rocas adquieren una magnetización remanente paralela a la dirección del campo magnético ambiental (el campo geomagnético). El segundo es que el campo geomagnético alterna entre estados de polaridad normal y polaridad inversa. La secuencia de cambios de polaridad define la escala global de polaridad geomagnética. Así es posible correlacionar la secuencia de cambios de polaridad grabada en una sección estratigráfica con la escala global.

\section{Marco geológico y estratigráfico}

En Coahuila se reconocen tres elementos paleogeográficos principales: el Bloque de Coahuila al sur, la Cuenca de Sabinas en la región central y la Plataforma Burros-Peyotes al norte (Figura 1). Además, hay algunos elementos positivos menores dentro de la Cuenca de Sabinas, como la Isla de La Mula (Jones et al., 1984) y la Isla de Monclova
(Eguiluz de Antuñano, 2001). La distribución de facies durante la mayor parte del Cretácico estuvo controlada por estos elementos (Wilson, 1990; Goldhammer, 1999). La secuencia estratigráfica de la región fue establecida originalmente por Burckhardt (1930) e Imlay (1940). El límite sur de la Cuenca de Sabinas se traza generalmente a lo largo de la FSM, aunque la parte más profunda de la cuenca se encuentra unos $50 \mathrm{~km}$ al norte en la región de la ciudad de Monclova.

En el VSM, localizado en la parte central de Coahuila, se encuentra expuesta la Falla San Marcos (Figura 2; McKee y Jones, 1979; Charleston, 1981; McKee et al., 1984; 1990). La FSM ha sido claramente documentada en el NE de México, tanto por evidencias estratigráficas (McKee et al., 1990) como geofísicas y estructurales (Charleston, 1981; Padilla y Sánchez, 1982; Chávez-Cabello et al., 2005, 2007; Gray et al., 2008). La falla tiene una longitud mínima de $300 \mathrm{~km}$ y una dirección $\sim \mathrm{N} 62^{\circ} \mathrm{W}$ (McKee et al., 1990). Pertenece a un grupo de lineamientos que han sido identificados o postulados para el NE de México como la Falla La Babia (Charleston, 1974, 1981), la zona de fractura Saltillo-Torreón (Murray, 1959), y el lineamiento TorreónMonterrey (De Cserna, 1970, 1976). La FSM limita a dos 


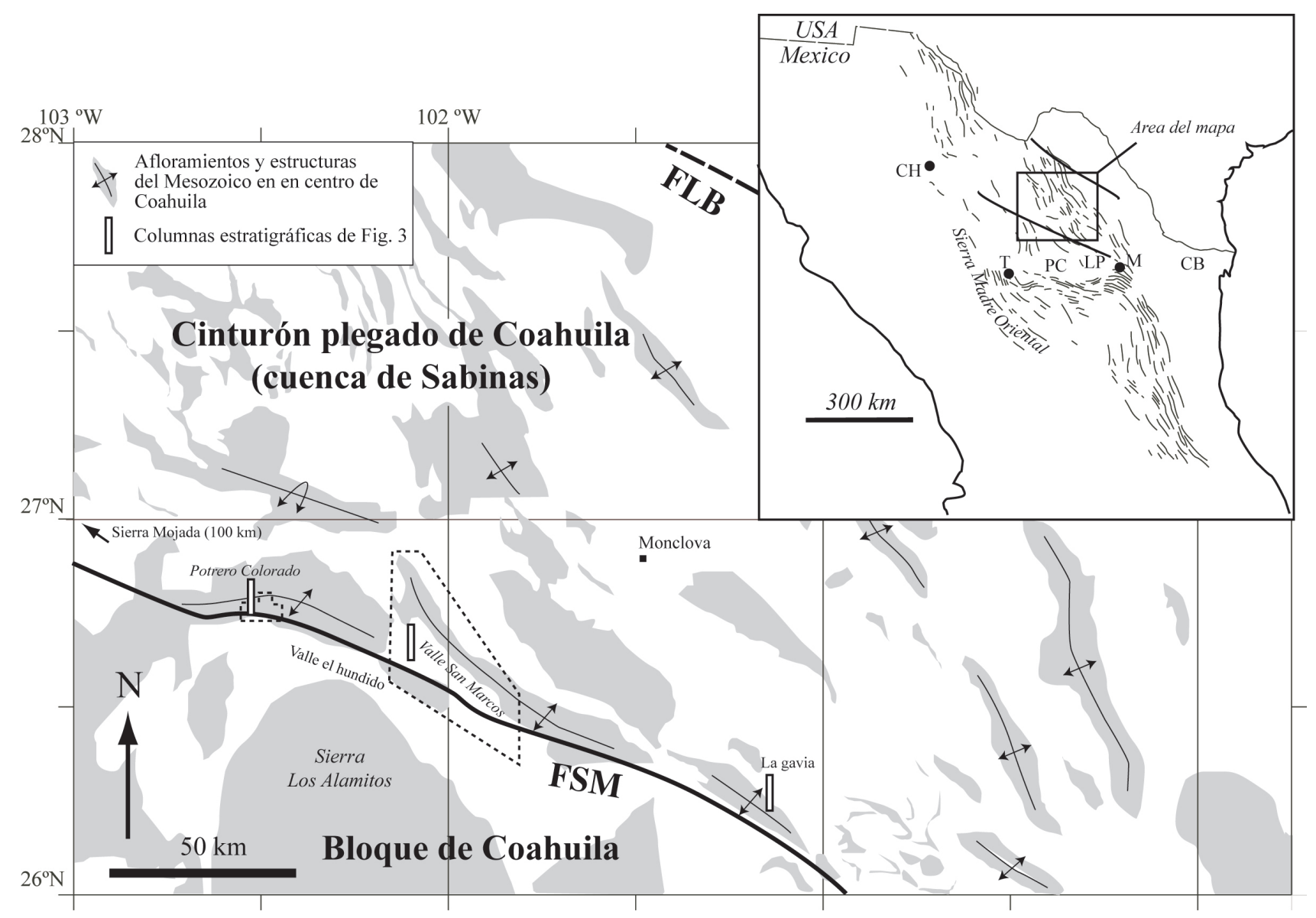

Figura 2. Localización de la Falla San Marcos, separando estructuralmente el Bloque de Coahuila y el Cinturón Plegado de Coahuila, en la parte central de Coahuila. También se muestran las áreas de muestreo (polígonos punteados), el Potrero Colorado y el valle de San Marcos. El recuadro muestra la ubicación del área del mapa en el contexto de las estructuras de la Sierra Madre Oriental. PC = Plataforma de Coahuila, LP = Cuenca La Popa, CB = Cuenca de Burgos; $\mathrm{CH}=$ Chihuahua; $\mathrm{T}$ = Torreón; $\mathrm{M}$ = Monterrey.

regiones contrastantes estructuralmente (Figura 2), al norte el Cinturón Plegado de Coahuila (CPC) y al sur el Bloque de Coahuila (BC).

McKee et al. (1990) interpretaron la parte inferior de la sección clástica del valle de San Marcos, de edad Jurásica, como una secuencia depositada bajo condiciones marinas (en sus primeros $2000 \mathrm{~m}$ de espesor), pero observaciones de Equisetum sp. en posición de crecimiento, grietas de desecación y estratificación cruzada de tipo cuchara muestran depósito en ambientes continentales, al menos para parte de la sección. La secuencia Jurásica fue dividida en tres unidades litoestratigráficas informales (McKee et al., 1990): capas Las Palomas, capas Sierra El Granizo, y capas Tanque Cuatro Palmas (Figura 3). Esta última representa facies litorales y lutitas de mar abierto del Titoniano, y es la única con contenido fósil diagnóstico. Las capas Tanque Cuatro Palmas son correlacionables con la Formación La Casita del Kimmeridgiano-Titoniano en el norte y NE de México. Por su posición entre dos unidades clásticas conglomeráticas continentales, las capas Tanque Cuatro Palmas representan una etapa de inactividad de la FSM
(McKee et al., 1990).

En el Cretácico Temprano, durante una primera reactivación con componente normal de la FSM se depositó al norte de esta falla la Formación San Marcos, con un espesor máximo de $\sim 1000 \mathrm{~m}$. Unidades correlacionables con ésta se depositaron al sur (Formación Carbonera) y al norte (Formaciones La Mula, Menchaca y Pátula) del Bloque de Coahuila. La Formación San Marcos se depositó bajo condiciones continentales y carece de fósiles con valor bioestratigráfico. Los detritos que componen a esta formación provienen del Bloque o Isla de Coahuila y de las capas de edad Jurásica que actuaron como fuente de detritos reciclados (McKee et al., 1990).

A la Formación San Marcos le sobreyace la Formación Cupido, compuesta por depósitos de una plataforma carbonatada bordeada por un franja de arrecife (Lehmann et al., 1999). En el área de estudio, la Formación Cupido está representada por depósitos intermareales y lagunares del interior de la plataforma (Lehmann et al., 1999). El contacto entre las Formaciones San Marcos y Cupido es transicional: la transición está marcada por areniscas y 


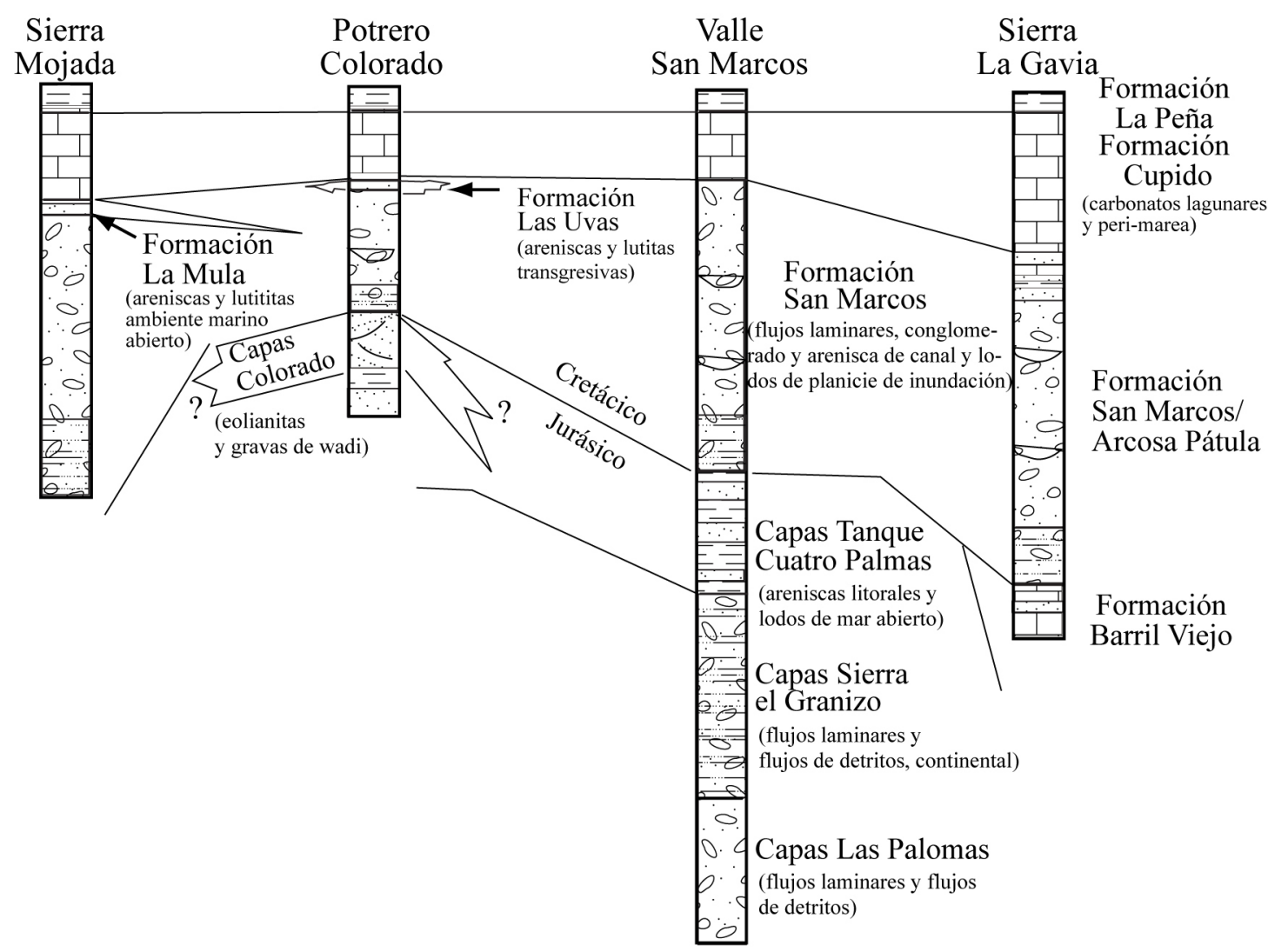

Figura 3. Estratigrafía del Jurásico-Cretácico a lo largo de la Falla de San Marcos, modificado de McKee et al. (1990).

limos intercalados, con tonalidades de color verde olivo. Estas facies son similares a las descritas para la Formación Las Uvas en el Bloque de Coahuila.

Durante el Terciario Temprano sobrevino el evento de deformación regional Laramide, que reactivó nuevamente a la FSM, con un comportamiento inverso que provocó el levantamiento del bloque al norte de la FSM y la inversión de la Cuenca de Sabinas. Por lo anterior, ocurrió el levantamiento de los conglomerados de las capas Las Palomas y sierra El Granizo, principalmente, los que se encuentran yuxtapuestos sobre rocas marinas calcáreas del Cretácico en el flanco NE de la sierra El Granizo, a lo largo de la traza de la FSM (McKee et al., 1988; Chávez-Cabello et al., 2005).

\subsection{La Formación San Marcos}

La litología característica de la Formación San Marcos corresponde a arcosa gruesa. Los granos de arena están pobremente clasificados y están soportados en una matriz de arena fina y limo. Las unidades conglomeráticas son comunes y muchos de los clastos están representados por gravas o guijarros de rocas volcánicas con algunos fragmentos de cuarzo, caliza y rocas plutónicas. En su parte inferior, en afloramientos al oriente del Potrero Colorado, son comunes, si no exclusivos, bloques de composición granodiorítica derivados directamente del basamento del BC que aflora sobre la traza de la FSM. Estos conglomerados muestran una burda estratificación, ocasionalmente estratificación gradada y estratificación planar. En la parte superior de la Formación San Marcos existen cuerpos ampliamente lenticulares de arenisca intercalados con depósitos finos. La secuencia en su parte media incluye varios ciclos de escala decamétrica con una clara tendencia granodecreciente hacia la cima. Los ciclos están compuestos por arenisca conglomerática en la base, horizontes tabulares de arenisca bien clasificada, limos y arcilla localmente alterada por pedogénesis en la cima. El contacto superior con la Formación Cupido es transicional, marcado por una alternancia de areniscas rojas y verdosas con limos, mostrando mejor clasificación que la secuencia inferior e interpretadas como facies litorales. El contacto con la unidad inferior está expuesto en el área de Potrero Colorado. Aquí sobreyace un paquete de arenisca roja de origen eólico asignado a las capas Colorado (informal) que McKee et al. (1990) correlacionan con las capas Tanque Cuatro Palmas por su posición estratigráfica, aunque las capas Colorado representan facies continentales de 
ambientes eólicos y de wadi, y no dunas costeras como sugieren McKee et al. (1990).

Los ambientes de depósito de la Formación San Marcos se interpretan como abanicos aluviales y bajadas (depósitos compuestos por flujos laminares y de detritos) en su parte inferior, con depósitos fluviales en su parte superior (McKee et al., 1990). Estos ambientes de depósito se encontraban sobre una región emergida en el interior de la plataforma Cupido según el modelo de Lehmann et al. (1999). González-Naranjo et al. (2008) sugieren que existió fallamiento activo durante los depósitos de, al menos, la parte inferior de la Formación San Marcos. La parte más alta de la Formación San Marcos es litológicamente similar a la Formación Las Uvas del Bloque de Coahuila y parece haberse depositado en ambientes transicionales. Así, la Formación San Marcos se hace claramente más fina hacia su parte superior, indicando una disminución en el relieve en la fuente y pendientes más suaves en los sistemas fluviales que alcanzan a desarrollar sistemas de meandros.

\section{Métodos de muestreo y laboratorio}

La Formación San Marcos se muestreó en dos localidades a lo largo de la Falla San Marcos (Figuras 4 y 5). La primera localidad se encuentra en el valle San Marcos (Figura 4), donde se obtuvieron 13 sitios en una secuencia de conglomerado, arenisca y lodolita roja fluvial de una secuencia de aproximadamente $100 \mathrm{~m}$ de espesor expuesta en una pequeña mesa dentro del valle. Por otro lado, en el Potrero Colorado se muestreó la secuencia inferior de la formación en la parte oriental del potrero (11 sitios) y la parte superior de la formación en la parte centro-occidental (11 sitios). La columna estratigráfica muestreada en la parte este de Potrero Colorado expone aproximadamente $150 \mathrm{~m}$ de facies de arenisca conglomerática con una estratificación burda de la Formación San Marcos, mientras que la sección de la parte centro-occidental es de aproximadamente $250 \mathrm{~m}$, pero por lo abrupto del terreno el intervalo de muestreo es más abierto. Las secciones del valle San Marcos y la parte centro-occidental de Potrero Colorado consisten en depósitos fluviales, alcanzando la sección de Potrero Colorado la transición con la Formación Cupido (facies Las Uvas). Cada sitio incluye de 5 a 7 muestras orientadas, y un sitio paleomagnético está restringido a un solo nivel estratigráfico (igual a una capa). El muestreo se hizo preferentemente en arenas de grano medio a fino. El intervalo promedio entre sitios es de aproximadamente $15 \mathrm{~m}$.

Las muestras paleomagnéticas fueron perforadas en campo usando una perforadora portátil y fueron orientadas in situ utilizando tanto brújula magnética como solar (con un error de orientación $\left.<1^{\circ}\right)$. En el laboratorio se prepararon especimenes estándares $(2.5 \mathrm{~cm}$ de diámetro y $2.1 \mathrm{~cm}$ de alto) para las mediciones de la magnetización remanente natural (MRN) y los procesos de desmagnetización. La

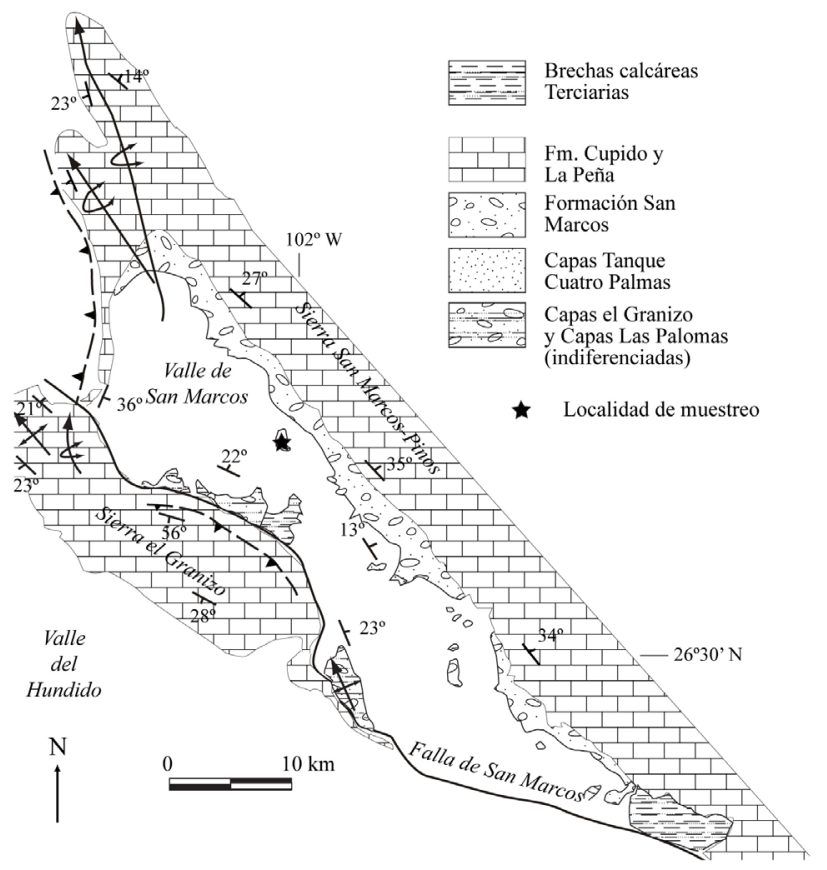

Figura 4. Mapa geológico del valle de San Marcos, mostrando la ubicación de la sección muestreada (modificado de Arvizu-Gutiérrez, 2006).

actitud de las capas fue determinada para cada una de las unidades estratigráficas para realizar las correcciones estructurales subsecuentes a los análisis.

Algunas muestras piloto fueron sujetas a desmagnetización progresiva de campos alternos (usando un desmagnetizador AF LDA-3A con tres capas de aislamiento con metal "mu"), y el resto fueron sujetas a desmagnetización térmica entre 8 y 20 pasos (usando un horno ASC TD-48SC). Se utilizaron inducciones máximas de $120 \mathrm{mT}$ y temperaturas de $680{ }^{\circ} \mathrm{C}$, respectivamente. Para la medición de la MRN se utilizó un magnetómetro de giro AGICO-JR-5A. Parte de las muestras fueron desmagnetizadas en el laboratorio de la Universidad de Nuevo México, E.U.A., utilizando un magnetómetro criogénico (2G-Enterprise Superconducting), un desmagnetizador AF (2G-Enterprise three-axis automated sample handler) y por último un desmagnetizador térmico (Schonstedt-D Furnace and $2 G$ Controllers).

La composición vectorial de la MRN fue analizada de forma visual mediante diagramas ortogonales de desmagnetización (Zijderveld, 1967) y las componentes de magnetización fueron determinadas usando el análisis de componentes principales (Kirschvink, 1980). Algunas direcciones medias fueron calculadas usando los análisis de trayectorias de círculos mayores (McFadden y McElhinny, 1988). Las direcciones promedio de cada uno de los sitios fueron calculadas usando las estadísticas de Fisher (1953). Algunos de los problemas encontrados durante el análisis fueron la desintegración de las muestras durante la desmagnetización, por lo que algunas muestras fueron 


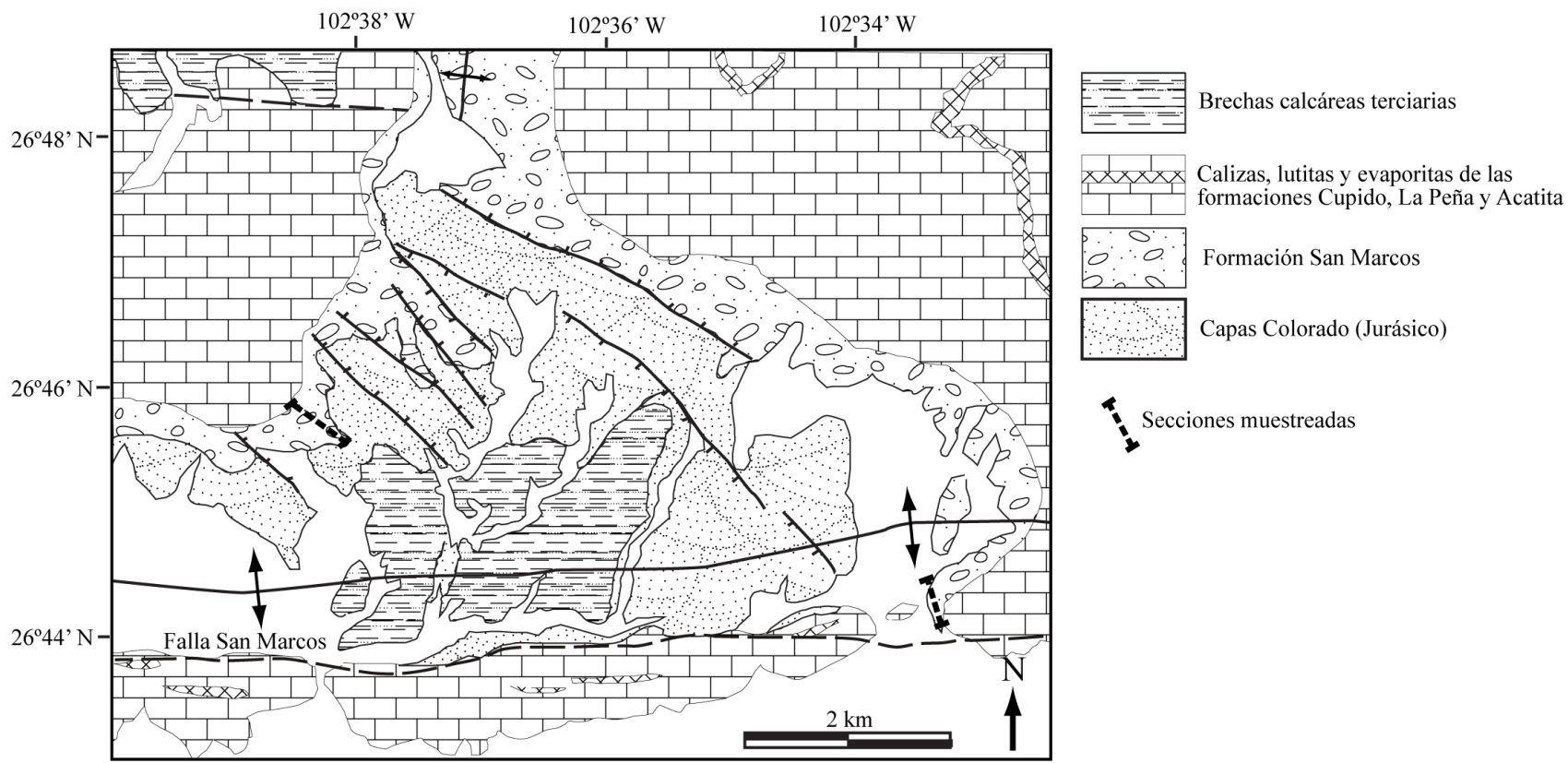

Figure 5. Mapa geológico del Potrero Colorado, mostrando la ubicación de las secciones muestreadas en los flancos este y oeste del Potrero. Modificada de González-Naranjo et al. (2008).

excluidas del cálculo final de la dirección media. Por otro lado, en ocasiones el número de muestras por sitio fue insuficiente para calcular una dirección media confiable y, finalmente, en pocos casos se observó un comportamiento inestable

\section{Resultados}

\subsection{Valle San Marcos}

La magnetización remanente natural de las rocas de la Formación San Marcos en el valle San Marcos es de una intensidad moderada (del orden de varios $\mathrm{mA} / \mathrm{m}$ ) y normalmente consiste de dos componentes vectoriales (Figura 6). La componente de baja temperatura de bloqueo (menor a $450^{\circ} \mathrm{C}$ ) tiene una dirección hacia el NNW o NNE y una inclinación moderadamente positiva; en cambio, la de alta temperatura se dirige hacia el NW con inclinación similar a la anterior o ligeramente más alta (Figura 6a). La dirección in situ promedio de la componente de baja temperatura es de $D=8.5^{\circ}, I=51.7^{\circ}(n=33$ muestras). Inducciones de $120 \mathrm{mT}$ no remueven una fracción significante de la MRN (Figura 6b). Pocas muestras fueron desmagnetizadas por campos alternos, pues en general este método no separa las magnetizaciones presentes en las muestras. En cambio la componente característica se define en un rango aproximado de $625-665^{\circ} \mathrm{C}$, lo que indica que la magnetización reside principalmente en hematita. La temperatura máxima de bloqueo es mayor a $665^{\circ} \mathrm{C}$. En algunas muestras se calcularon trayectorias de círculos mayores para determinar la dirección de magnetización característica (Figura 6c). Estas muestras corresponden a direcciones de polaridad inversa, dirigidas al SE y con inclinaciones negativas (Figura 6e). Un gran número de muestras están afectadas por descargas eléctricas (rayos), las cuales se identifican como magnetizaciones de baja estabilidad (baja coercitividad) y direcciones erráticas. Estas magnetizaciones secundarias son fácilmente eliminadas durante el proceso de desmagnetización.

En un sitio es posible reconocer 3 componentes de la MRN, el sitio FSM 5 (Figuras 6d y 6e). De hecho, el sitio FSM 5 tiene direcciones de la magnetización característica de ambas polaridades; después de remover una magnetización secundaria dirigida al Norte y de inclinación positiva, es posible identificar componentes de polaridad inversa entre $300^{\circ}$ y $665^{\circ} \mathrm{C}$ y polaridad normal entre $665^{\circ}$ y $680^{\circ} \mathrm{C}$. Los sitios FSM 1, FSM 5 y FSM 13 (Figura 6e) dentro de la Formación San Marcos registran entonces, en algunos de sus especímenes, magnetizaciones de polaridad inversa (Tabla 1). Cerca del 50\% de las muestras obtenidas fueron útiles en los análisis paleomagnéticos. Estas muestras definen direcciones características o círculos mayores con valores MAD (maximum angular deviation) menores a $10^{\circ}$. La dirección media para la componente característica de esta localidad, corregida estructuralmente, es de $D=346$ ${ }^{\circ}, I=49.6^{\circ}, N=5$ sitios, $k=105.7, \alpha_{95}=7.5^{\circ}$ (Tabla 1$)$.

\subsection{Potrero Colorado}

La magnetización remanente natural de las muestras del Potrero Colorado ha sido descrita en una publicación 


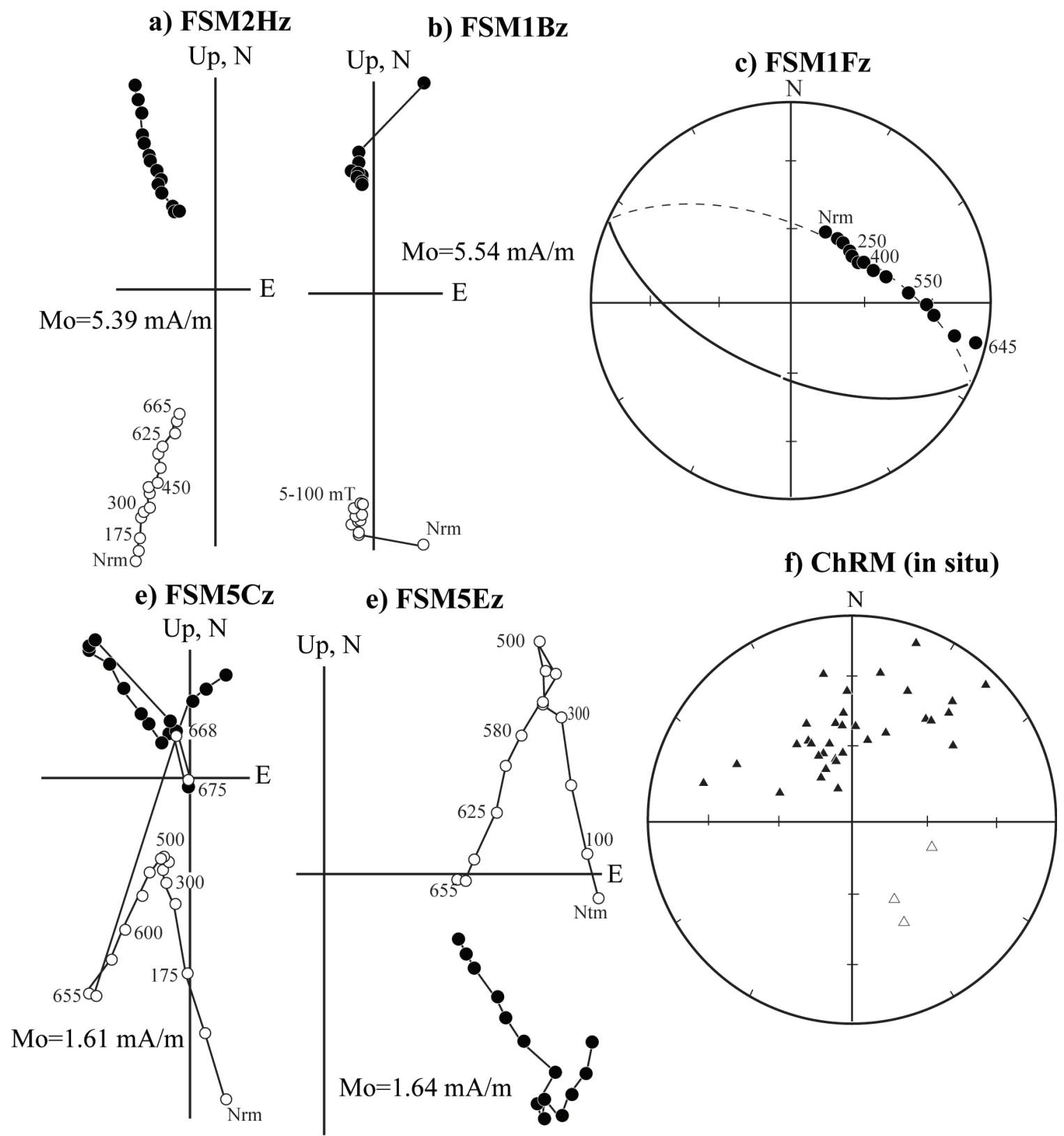

Figura 6. a), b), d) y e) Diagramas de desmagnetización ortogonales de muestras representativas de la Formación San Marcos. La temperatura está dada en grados Celsius $\left({ }^{\circ} \mathrm{C}\right)$ y las inducciones en militeslas $(\mathrm{mT})$. c) Ejemplo de una trayectoria de desmagnetización de un círculo mayor de la muestra FSM1F(z). f) Proyección estereográfica mostrando las direcciones de las muestras obtenidas in situ de la Formación San Marcos. Los triángulos rellenos son direcciones de polaridad normal y los triángulos huecos son direcciones de polaridad inversa (Arvizu-Gutiérrez, 2006).

reciente (González-Naranjo et al., 2008), por lo que sólo los rasgos más sobresalientes se resumen en los siguientes párrafos. La MRN tiene intensidades moderadas $\left(10^{-3}\right.$ $\mathrm{A} / \mathrm{m}$ ), al igual que las del valle San Marcos, y también se reconocen dos componentes de magnetización: una de baja temperatura, con temperaturas máximas de bloqueo en el laboratorio entre $580^{\circ}$ y $600^{\circ} \mathrm{C}$, y otra de alta temperatura, la cual se remueve por completo cerca de $\operatorname{los} 680^{\circ} \mathrm{C}$ (Figuras 7 y 8 ).

Existe un gran número de muestras en las cuales no es posible aislar la componente de alta temperatura mediante la desmagnetización térmica, ya que a temperaturas $>600{ }^{\circ} \mathrm{C}$ el comportamiento de las muestras es inestable. Es por ello que se optó por utilizar el método de trayectorias de círculos mayores para determinar la componente característica (González-Naranjo et al., 2008). La polaridad de la magnetización es, sin embargo, fácilmente interpretable a partir de las trayectorias de círculos mayores. Para los cálculos finales solo fueron utilizados 7 sitios para el flanco oriental y 6 sitios para el occidental. 
Tabla 1. Resumen de datos paleomagnéticos para la Formación San Marcos.

\begin{tabular}{cccccc}
\hline Localidad & Declinación & Inclinación & Sitios, $\boldsymbol{k}, \boldsymbol{\alpha}_{\mathbf{9 5}}$ & Referencia & $\mathbf{R}+/-\Delta \mathbf{R}$ \\
\hline Valle San Marcos & $346.0^{\circ}$ & $49.6^{\circ}$ & $5,105.7,7.5^{\circ}$ & Este estudio & $5.8 \pm 12.1$ \\
Potrero Colorado E & $192.0^{\circ}$ & $-53.3^{\circ}$ & $7,43.6,9.2^{\circ}$ & 1 & $30.8 \pm 12.8$ \\
Potrero Colorado W & $333.6^{\circ}$ & $58.0^{\circ}$ & $6,28.3,12.8^{\circ}$ & 1 & $-4.6 \pm 17.7$ \\
Over all mean & $352.7^{\circ}$ & $55.5^{\circ}$ & $18,27.1,6.8^{\circ}$ & 1 & $11.5 \pm 11.9$ \\
North America craton & $341.2^{\circ}$ & $51.8^{\circ}$ & $2.1^{\circ}$ & 2 & \\
\hline
\end{tabular}

${ }^{1}$ Direcciones recalculadas de González-Naranjo et al . (2008)

${ }^{2}$ McElhinny y McFadden, 2000. Anomalías de inclinación son insignificantes

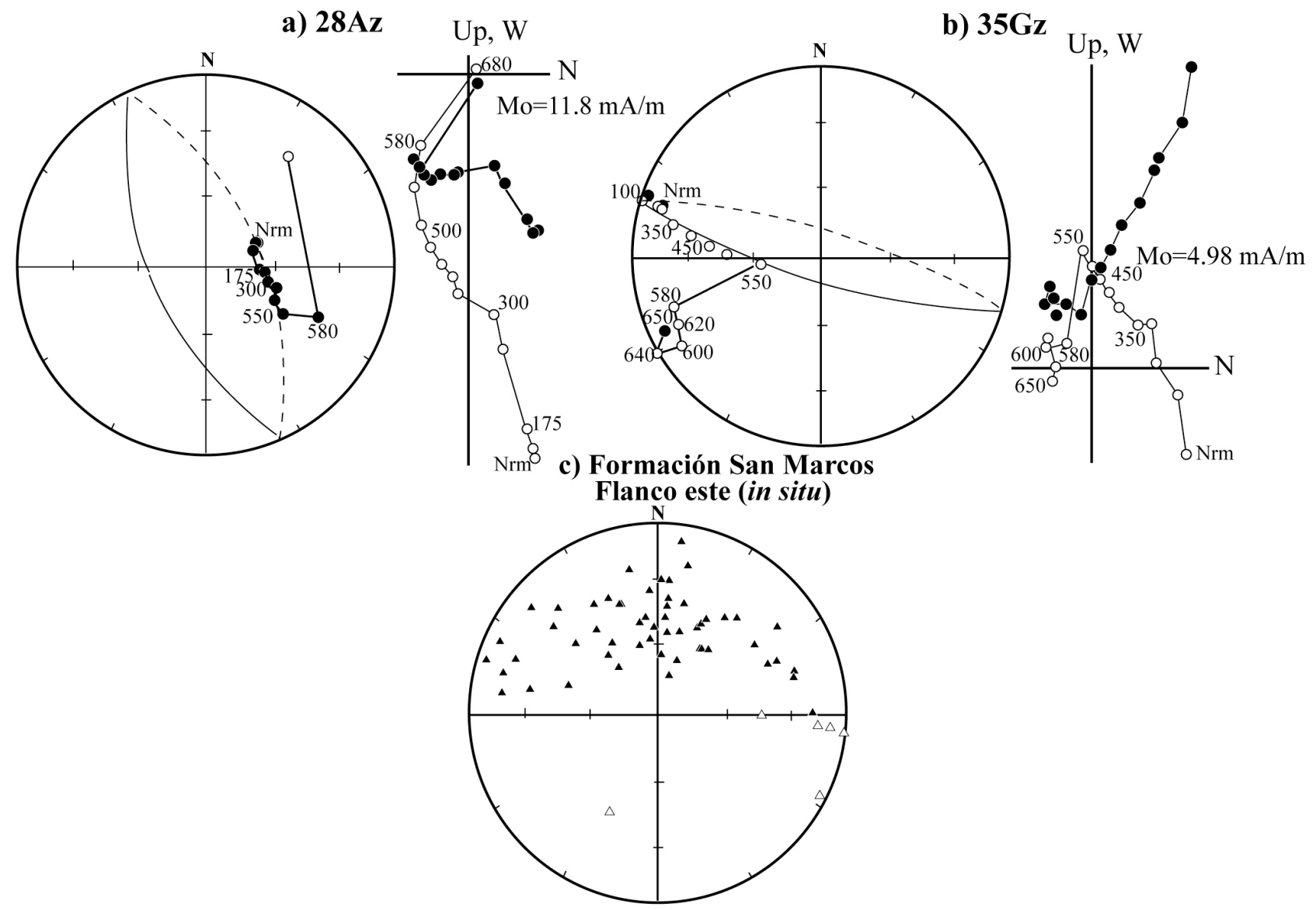

Figura 7. a) y b) Diagramas de desmagnetización (proyección ortogonal y proyección estereográfica) de muestras representativas de la Formación San Marcos en el flanco oriental de Potrero Colorado (González-Naranjo, 2006). La temperatura está dada en grados Celsius $\left({ }^{\circ} \mathrm{C}\right)$ y las inducciones en militeslas (mT). a) Ejemplo de una trayectoria de desmagnetización de un círculo mayor de la muestra 28Az. b) Diagrama de desmagnetización ortogonal de componentes. c) Proyección estereográfica mostrando los polos a círculos mayores (in situ) de muestras de la Formación San Marcos en el flanco oriental. Los triángulos rellenos son proyecciones en el hemisferio inferior y los triángulos huecos son proyecciones en el hemisferior superior.

\section{2. 1 Flanco Oriental}

La mayoría de las muestras del flanco oriental tienen un comportamiento uniforme durante la desmagnetización, definiendo trayectorias de círculos mayores. La magnetización de más baja temperatura es espuria y se remueve cerca de los $300{ }^{\circ} \mathrm{C}$. Una magnetización de temperatura intermedia de bloqueo se remueve entre 300 ${ }^{\circ}$ y $500{ }^{\circ} \mathrm{C}$ y se dirige hacia el N-NE con una inclinación positiva (Figura 7a y 7b). La dirección in situ promedio de 

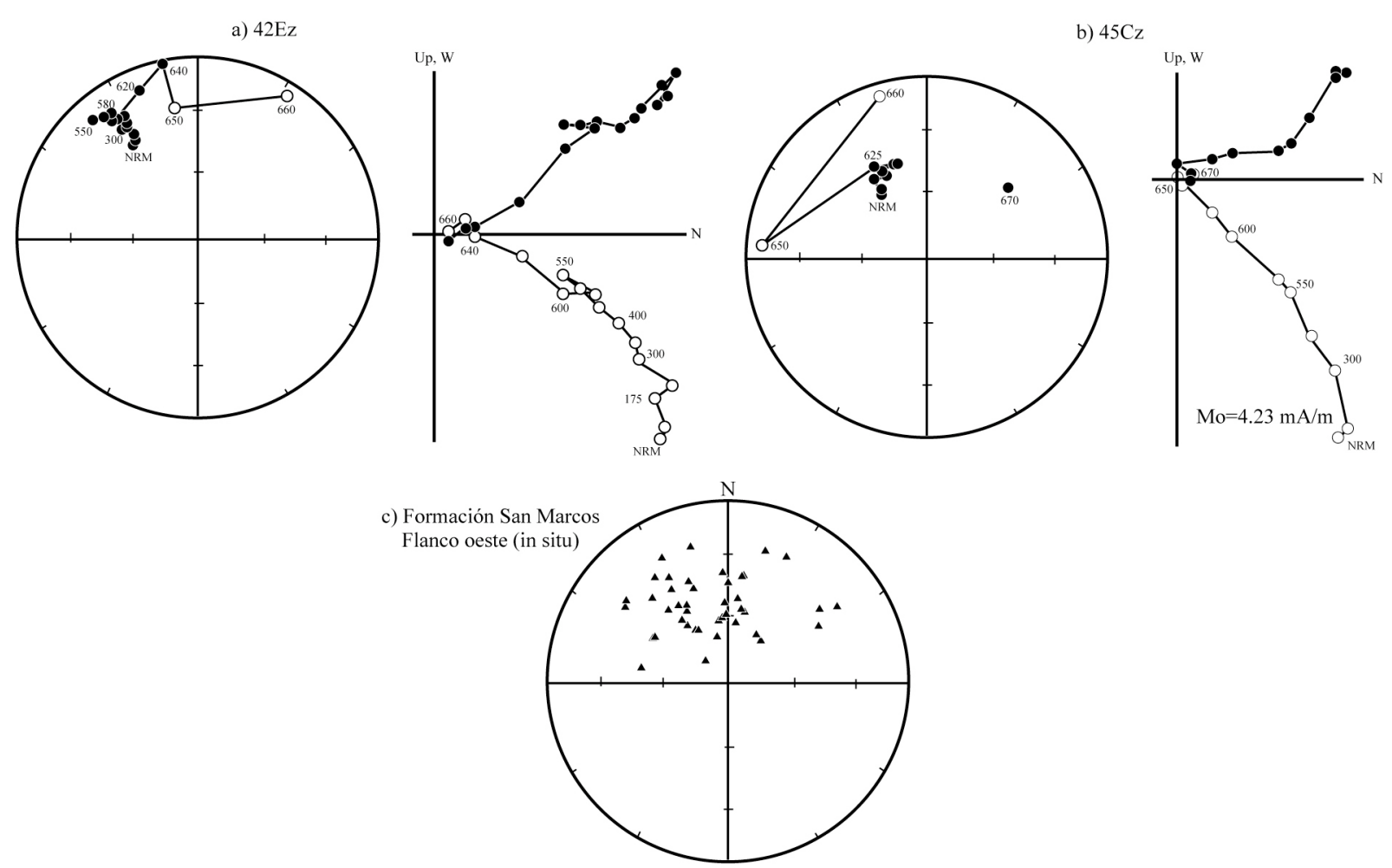

Figura 8. a) y b) Diagramas de desmagnetización ortogonales y proyección estereográfica de muestras representativas de la Formación San Marcos en el flanco centro-occidental de Potrero Colorado (González-Naranjo, 2006). La temperatura está indicada en grados Celsius $\left({ }^{\circ} \mathrm{C}\right)$ y las inducciones en militeslas (mT). a) Ejemplo de una trayectoria de desmagnetización de un círculo mayor de la muestra 42Ez. b) Diagrama de desmagnetización ortogonal de componentes. c) Proyección estereográfica mostrando las direcciones de las muestras obtenidas in situ de la Formación San Marcos en el flanco centrooccidental. Los triángulos rellenos son direcciones de polaridad normal y los triángulos huecos son direcciones de polaridad inversa.

la componente de temperatura intermedia es de $D=1.7^{\circ}, I$ $=43.9^{\circ}(n=51$ muestras $)$. En cambio la componente de alta temperatura, véase el ejemplo en la muestra $35 \mathrm{Gz}$ (Figura $7 b)$, no se define en el proceso de desmagnetización y por ello se calcularon trayectorias de círculos mayores para determinarla. En los ejemplos, en redes estereográficas, se pueden observar claras trayectorias de desmagnetización que definen círculos mayores con buena precisión (MAD $<10^{\circ}$ ). A bajas temperaturas la dirección resultante se encuentra en el hemisferio norte y posteriormente a altas temperaturas migra hacia el hemisferio sur con trayectorias hacia inclinaciones negativas (Figura 7b). La dirección media de esta localidad, corregida estructuralmente, para la componente característica es de $D=192.0^{\circ}, I=-53.3^{\circ}, N=$ 7 sitios, $k=43.6, \alpha_{95}=9.2^{\circ}$ (Tabla 1 ). Se considera que el comportamiento inestable a altas temperaturas se debe a la alteración de fragmentos líticos presentes en las muestras.

\subsubsection{Flanco Occidental}

En el flanco occidental del área de Potrero Colorado (Figura 5), casi todas las muestras tienen una dirección hacia el NW; esto puede observarse en la muestra $42 \mathrm{Ez}$ (Figura 8a). Las inclinaciones varían de intermedias a altas con un comportamiento relativamente estable de las direcciones durante el experimento de desmagnetización.
Ocasionalmente, la trayectoria de desmagnetización observada entre $580^{\circ}$ y $640{ }^{\circ} \mathrm{C}$ no está dirigida al origen (Figura $8 \mathrm{a}$ y $8 \mathrm{~b}$ ), por lo que la dirección característica se calculó sin incluir las temperaturas $>640^{\circ} \mathrm{C}$. La dirección media característica corregida estructuralmente es de $D=$ $333.6^{\circ}, I=58.0^{\circ}, N=6$ sitios, $k=28.3, \alpha_{95}=12.8^{\circ}$ (Tabla 1 ).

La magnetización remanente que reside en hematita en rocas sedimentarias de ambientes continentales puede tener un origen químico o detrítico. Una magnetización de temperatura alta y de rango discreto de bloqueo (por ejemplo, $650^{\circ}$ a $680^{\circ} \mathrm{C}$ ) se asocia generalmente a hematita especular que puede tener origen detrítico (Larson y Walker, 1982). De igual manera, una magnetización de temperaturas menores y distribuidas (por ejemplo, $300^{\circ}$ a $620^{\circ} \mathrm{C}$ ) se asocia generalmente a hematita pigmentaria de grano fino (cuyo origen es químico). El comportamiento de las muestras de la Formación San Marcos sugiere contribuciones de ambas fuentes, hematítica detrítica y química.

\section{Correlación con la Escala de Polaridad Geomagnética}

Las zonas de polaridad identificadas en cada una de las columnas estratigráficas muestreadas son comparadas con 
la Escala de Polaridad Geomagnética de Gradstein et al. (2005) en la Figura 9. Para construir la zonación de polaridad magnética, el límite entre dos zonas de distinta polaridad se establece en un punto intermedio entre sitios adyacentes con polaridad distinta. La presencia de dos polaridades en un mismo sitio se interpreta como el registro de intervalos de polaridad de corta duración comparados con el tiempo de adquisición de una magnetización química. Una fracción de hematita de origen químico puede, por ejemplo, grabar la polaridad dominante (normal), mientras que sólo la fracción detrítica graba con fidelidad el corto intervalo de polaridad inversa. Tal es el caso por ejemplo de magnetizaciones inversas en el sitio FSM 5 (Figuras 6d y 6e), donde la polaridad normal se interpreta como adquirida en un tiempo posterior al depósito durante el intervalo de polaridad dominantemente normal del Aptiano. Esta observación es común cuando ocurren eventos cortos de polaridad durante el proceso de adquisición de la remanencia en lechos rojos
(Larson y Walker, 1982).

En el flanco este del Potrero Colorado se muestreó la parte basal de la Formación San Marcos que en esta zona se caracteriza por presentar conglomerado de guijarros y arenisca de grano grueso a medio, con escasas intercalaciones de limonita café-rojiza (Figura 9). Esta sección se encuentra a pocos metros del contacto con el basamento expuesto del BC. Dentro de la columna muestreada $(100 \mathrm{~m})$ se identificaron dos zonas de polaridad normal (sitios 32n, 37 y 38; Figura 9) además de una zona de aparente larga duración definida por 7 sitios de polaridad inversa (28-31 y 33-36). Las zonas se numeraron de la base a la cima, usando pares para los intervalos normales y añadiendo el prefijo PO (Figura 9). La presencia de polaridades inversas confirma que la sección es del Aptiano o más antigua, es decir, anterior al Largo Intervalo Normal del Cretácico (Opdyke y Channell, 1996).

En la localidad en la parte norte del valle de San Marcos,

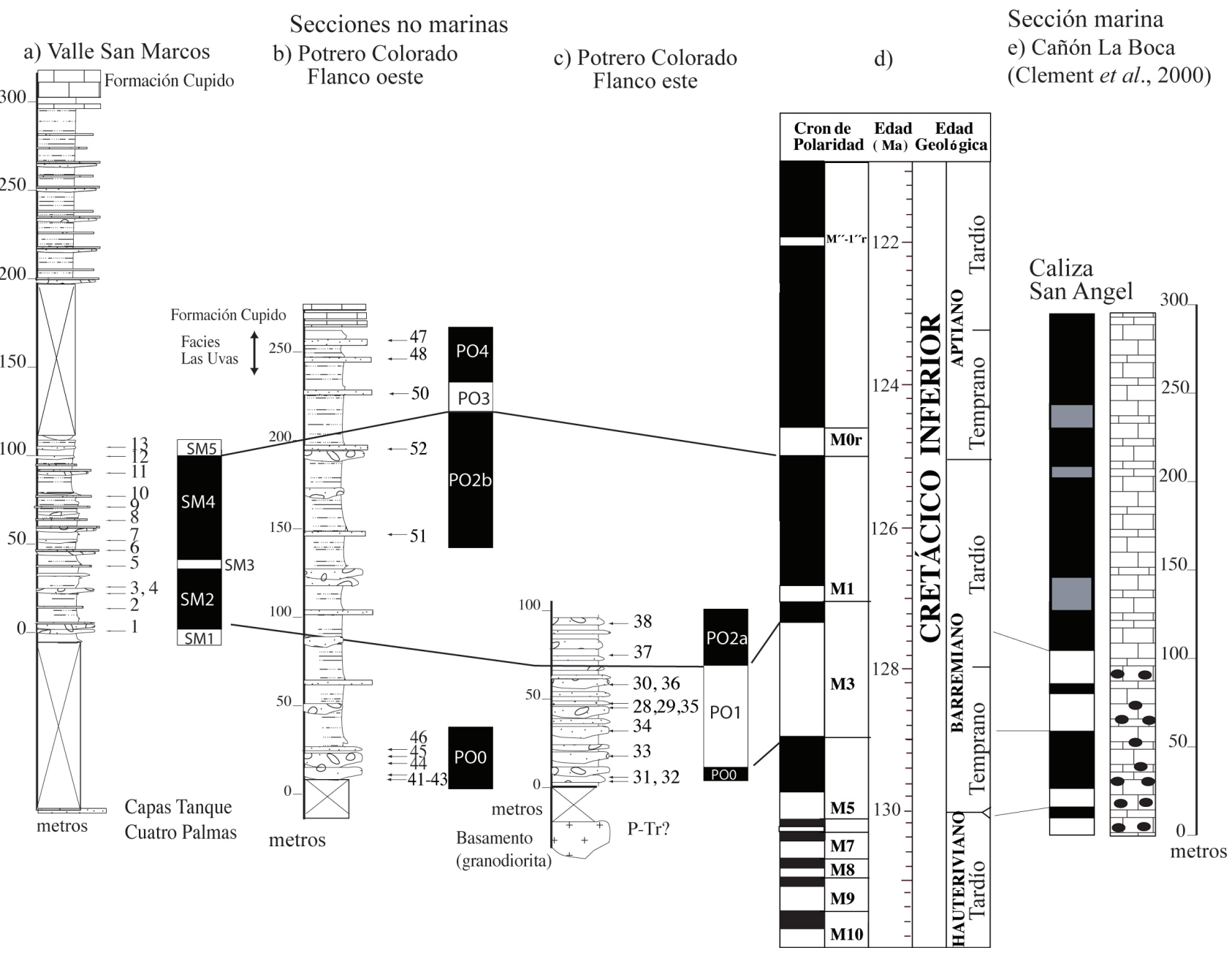

Figura 9. Litología y magnetoestratigrafía de la Formación San Marcos en las columnas muestreadas en el valle de San Marcos y Potrero Colorado. a) Columna estratigráfica esquemática muestreada en el valle de San Marcos ilustrando los sitios de muestreo. b) Columna del flanco centro-occidental de Potrero Colorado mostrando los sitios paleomagnéticos. c) Columna del flanco oriental de Potrero Colorado mostrando los sitios paleomagnéticos. d) Escala del Tiempo de Polaridad Geomagnética utilizada para la comparación e identificación de las zonas de polaridad reconocidas en las columnas muestreadas (Gradstein et al., 2005). e) Magnetoestratigrafía de la Caliza San Ángel (Clement et al., 2000) 
donde se colectaron 15 sitios en la Formación San Marcos, el muestreo se realizó en parte de la columna que aflora de manera continua por aproximadamente $100 \mathrm{~m}$, pero no aflora la base de la sección. La sección muestreada se encuentra en la parte NW del valle San Marcos (Figura 4), por lo que representa la parte media de la formación. La base de la sección está cubierta, pero aflora a unos 15 $\mathrm{km}$ al este, cerca de la localidad conocida como Loma El Cuevudo, donde sobreyace a las capas Tanque Cuatro Palmas. La posición de la columna muestreada respecto a estos afloramientos y la actitud de las capas sugieren que no es un espesor considerable el que subyace a la columna muestreada. Este espesor se estima en $\sim 100 \mathrm{~m}$. En la localidad de muestreo se aprecia la alternancia de conglomerado y arenisca gruesa con limonita y arenisca fina; conforme se asciende estratigráficamente va disminuyendo el conglomerado (Figura 9). Los datos obtenidos muestran un periodo de polaridad normal relativamente largo entre dos polaridades inversas, intervalos marcados por los sitios FSM 1 y FSM 13 (Figura 9), e interrumpido por un corto evento de polaridad inversa (sitio FSM 5). Las zonas de polaridad se numeraron de la base a la cima, con el prefijo SM (Figura 9).

La columna estratigráfica muestreada en el flanco oeste del Potrero Colorado fue de aproximadamente $300 \mathrm{~m}$ (González-Naranjo et al., 2008), donde se muestrearon 11 sitios que cubrieron la mayor parte de la columna (Figura 9), la cual incluye a la parte más superior de la Formación San Marcos en ésta localidad. La sección tiene una litología general de areniscas-lutitas y conglomerados que hacia la parte superior es transicional a la Formación Cupido con la presencia de areniscas, limolitas y horizontes limo-arcillosos de color verdoso intercalados con calizas en la parte más alta de la secuencia (Figura 9), que se interpretan como las facies de la Formación Las Uvas. Solamente se encontró un sitio de polaridad inversa (50), casi en la cima de la columna, y el resto fueron sitios de polaridad normal en los sitios 41-49, 51 y 52. La zona de polaridad se numeró continuando con la zonación del flanco este de Potrero Colorado.

La correlación con la escala magnética de polaridad es relativamente simple. La zonación en la Formación San Marcos está dominada por polaridad normal. Además, los intervalos de polaridad inversa son relativamente cortos en duración comparados a los intervalos de polaridad normal. Solamente la zona PO1, en la sección este de Potrero Colorado corresponde a un intervalo relativamente largo (por su espesor). Esas características son típicas de la zonación del intervalo Barremiano-Aptiano, anterior al Largo Intervalo Normal del Cretácico. La zona de polaridad normal en la posición más baja de la columna se correlaciona con la parte superior del cron de polaridad M5n, mientras que el resto de las zonas de polaridad inversa corresponderían a los crones de polaridad M3r, M1r y M0r (zonas SM1, SM3 y SM5). El intervalo del extremo superior, en la sección centro-oeste de Potrero Colorado y dentro de las facies Las Uvas, subyace a la zona de polaridad normal
M"-1"r. Por lo tanto, se le puede asignar a la columna muestreada una edad del Barremiano Temprano al Aptiano Temprano (131-125 Ma; Gradstein et al., 2005).

Cabe notar que la correlación con los crones de la secuencia del Hauteriviano es poco probable, pues en ella los intervalos de polaridad normal e inversa son de aproximadamente la misma duración (M6 a M10). Igualmente, la correlación con la secuencia del Valanginiano está más bien caracterizada por la predominancia de polaridad inversa con intervalos cortos de polaridad normal y tampoco asemeja a la secuencia reconocida en la Formación San Marcos. Existe una correlación relativamente buena entre la secuencia del Berriasiano y parte de la secuencia de la Formación San Marcos, pero considerando la edad Aptiana para la parte alta de la Formación Cupido, el contacto transicional entre las Formaciones San Marcos y Cupido y el dominio de polaridades normales en la secuencia superior, una edad del Berriasiano es de probabilidad baja.

Si se interpreta el intervalo de polaridad normal en las capas basales de la Formación San Marcos como del Barremiano Temprano, no hay necesidad de hacer variar la tasa de sedimentación de forma notable dentro de la sección para correlacionar el resto de ella a la escala geomagnética de polaridad. Así, si consideramos por ejemplo que la sección en el valle de San Marcos se depositó principalmente durante el cron M1, con una duración de aproximadamente $3 \mathrm{Ma}$, podemos determinar la tasa de sedimentación de la Formación San Marcos durante la reactivación de la FSM en el Cretácico Temprano, siendo de aproximadamente $33.3 \mathrm{~m} / \mathrm{Ma}$. Por supuesto, esto no quiere decir que la tasa de acumulación sea constante; la interpretación correcta es que los cambios en dicha tasa no son grandes durante el período de acumulación.

La correlación obtenida con la escala magnética de polaridad global significa que durante el depósito de la Formación San Marcos hubo una tasa de sedimentación relativamente baja en comparación a las que existen para ambientes extensionales y de tectónica activa típicos. Se concluye que la depositación de la Formación San Marcos se debió principalmente a una subsidencia diferencial entre el Bloque de Coahuila y la Cuenca de Sabinas durante el Cretácico Temprano producto de los ajustes isostáticos en ese tiempo, que resultaron en reactivación de la falla. La tasa de sedimentación de la Formación San Marcos en el Potrero Colorado es similar a la del valle San Marcos, pero ligeramente menor.

La Figura 9 también muestra la correlación entre la secuencia de polaridad en la Formación San Marcos y la sección marina de cuenca de la Caliza San Ángel, en el área de Monterrey (Clement et al., 2000). Esta sección comprende la secuencia que subyace a la Formación La Peña, del Aptiano Tardío. La Caliza San Ángel es ligeramente más antigua, extendiendo su rango estratigráfico hasta el Hauteriviano. Para las facies de plataforma del Barremiano-Aptiano (Formación Cupido) no existen datos magnetoestratigráficos. Esta secuencia 
fue generalmente remagnetizada durante la deformación de finales del Cretácico y principios del Terciario. Sin embargo, la estratigrafía física y los datos bioestratigráficos y quimioestratigráficos presentados por Lehmann et al. (1999) permiten incorporar el sistema de la Plataforma Cupido en el diagrama cronoestratigráfico de la Figura 1. La presencia de Globigerinelloides algerianus y Dufrenoya sp. en la Formación La Peña indica una edad del Aptiano Tardío (Lehmann et al., 1999). Además, dentro de la Formación Cupido, estos autores reportan el amonoideo Eodesmoceras sp. y los foraminíferos Neotrocholina sp. y Vercorsella sp., del Barremiano. Con base en esto, el rango estratigráfico es claramente del Barremiano al Aptiano Temprano.

Los datos magnetoestratigráficos sugieren que las facies fluviales de baja energía en la Formación San Marcos y las facies transgresivas de línea de costa de la Formación Las Uvas, presentes en su cima, se depositaron durante la fase de inundación que causaron la retrogresión y retroceso (backstep) de la plataforma Cupido, propuestos por Lehmann et al. (1999). Este evento de inundación es diacrónico y está marcado por la unidad transgresiva Cupidito (Wilson y Pialli, 1977); de esta manera, los ambientes marinos alcanzan el margen norte de la Paleo-isla de Coahuila en Potrero Colorado en el Aptiano Temprano (como muestra la estratigrafía física anterior al depósito de la Formación La Peña).

\section{Implicaciones tectónicas}

La Tabla 1 resume las direcciones medias obtenidas para las localidades muestreadas en la Formación San Marcos. Las localidades en el Potrero Colorado fueron reportadas por González-Naranjo et al. (2008). En ese trabajo se interpretan las direcciones discordantes en la base de la sección (flanco oriental del Potrero Colorado), como producto de la deformación asociada a la reactivación de la FSM. Los sitios en esa sección indican una rotación horaria, y se propuso un modelo con un relevo entre segmentos de la FSM para explicar la rotación. Los datos para la secuencia más joven de la formación son concordantes, por lo que se propone que la actividad de la falla cesó durante el depósito de la Formación San Marcos.

Para la localidad en el valle de San Marcos la dirección media, aunque definida por un número pequeño de muestras útiles, sugiere también la presencia de una rotación horaria de $5.8^{\circ} \pm 12.1^{\circ}$, pero ésta no es estadísticamente significativa. Considerando que rocas del Jurásico Superior en el valle de San Marcos, al pie de la sierra El Granizo, son concordantes con la dirección de referencia para el cratón de Norte América (Arvizu-Gutiérrez, 2006), interpretamos la rotación observada en la Formación San Marcos como concordante. Sin embargo, una pequeña rotación local relacionada a la reorientación aparente del eje del anticlinal de la sierra de San Marcos-Pinos en su terminación occidental no puede descartarse completamente (Figuras 2 y 4 ). Los pliegues a lo largo de la FSM en esta región (sierra la Fragua, valle de San Marcos y la Gavia) se han interpretado como pliegues por inversión de falla de basamento durante la inversión de la Cuenca de Sabinas (y reactivación de la FSM; ChávezCabello, 2005).

\section{Conclusiones}

La Formación San Marcos fue muestreada en dos localidades a lo largo de la falla San Marcos donde se obtuvieron 3 columnas estratigráficas representativas de la formación (parte basal, media y superior). Se reconocieron dos componentes principales: una de baja y otra de alta temperatura, ambas dirigidas hacia el N-NW. La componente característica se observa en un rango de temperaturas de bloque entre $625^{\circ}$ y $660^{\circ} \mathrm{C}$, lo cual sugiere que la magnetización reside en hematita. La dirección media de la componente característica es de $D e c=352.7$ ${ }^{\circ}$, Inc $=55.5^{\circ}, N=3$ localidades (18 sitios), $k=27.07$, $\alpha_{95}=6.8^{\circ}$. La dirección media es discordante, pero los datos sugieren que existieron rotaciones relativas entre las localidades muestreadas y que las rotaciones son respecto a ejes verticales locales.

La magnetoestratigrafía general realizada en la Formación San Marcos proporciona una edad más precisa de ésta formación dentro del Cretácico Temprano, la cual es de una edad del Barremiano Temprano al Aptiano Temprano (131-125 Ma). Asumiendo que la tasa de sedimentación es relativamente uniforme, ésta es relativamente baja (33$48 \mathrm{~m} / \mathrm{Ma}$ ) comparada con lo que ocurre comúnmente en los ambientes extensionales activos. La depositación de la Formación San Marcos se debió principalmente a una subsidencia diferencial entre el Bloque de Coahuila y la Cuenca de Sabinas durante el Cretácico Temprano, producto de ajustes isostáticos que dispararon la reactivación de la Falla de San Marcos.

\section{Agradecimientos}

Agradecmos al Dr. José Jorge Aranda Gómez por la beca otorgada por la cual IAG realizó la campaña de muestreo en el marco proyecto CONACyT clave 47401-T. RMG agradece el apoyo del proyecto PAPIIT-UNAM IN1210023. Agradecemos las cuidadosas revisiones de Tim Lawton y Konstantin Krivosheya, así como el trabajo de los editores de este volumen.

\section{Referencias}

Arvizu-Gutiérrez, I.R., 2006, Paleomagnetismo de rocas jurásicas y cretácicas del Valle San Marcos, Coahuila, México: Juriquilla, Querétaro, Centro de Geociencias, Tesis de Maestría, 135 p.

Burckhardt, C., 1930, Etude synthétique sur le Mésozoique mexicaine: Mémoires de la Societé Paléontologique Suisse, 49, 1-123. 
Charleston, S., 1974, Stratigraphy, tectonics and hydrocarbon potential of the Lower- Cretaceous, Coahuila series, Coahuila, Mexico: Ann Arbor, Michigan, E.U.A., University of Michigan, Tesis doctoral, $268 \mathrm{p}$.

Charleston, S., 1981, A summary of the structural geology and tectonics of the State of Coahuila, Mexico, en Schmidt, C.I., Katz, S.B. (eds.) Lower cretaceous stratigraphy and structure, northern Mexico: West Texas Geological Society Publication, Field Trip Guidebook, 81-74, 28-36.

Chávez-Cabello, G., 2005, Deformación y magmatismo cenozoicos en el Sur de la Cuenca de Sabinas, Coahuila, México: Juriquilla, Querétaro, Centro de Geociencias, Universidad Nacional Autónoma de México, Tesis doctoral, $226 \mathrm{p}$.

Chávez-Cabello, G., Aranda-Gómez, J.J., Molina-Garza, R., Cossío-Torres, T., Arvizu-Gutiérrez, G., González-Naranjo, G.A., 2007, The San Marcos fault: A Jurassic multi-reactivated basement structure in northeastern Mexico, en Alaniz-Álvarez, S.A., Nieto-Samaniego, A.F. (eds.), Geology of México: Celebrating the Centenary of the Geologial Society of México, Geological Society of America Special Paper, 422, 261-286.

Chávez-Cabello, G., Aranda-Gómez, J.J., Molina-Garza, R.S., CossíoTorres, T., Arvizu-Gutiérrez, I.R., González-Naranjo, G.A., 2005, La Falla San Marcos: una estructura jurásica de basamento multirreactivada del noreste de México: Boletín de la Sociedad Geológica Mexicana, 57, 27-52.

Clement, B.M., Poetisi, E., Bralower, T.J., CoBabe, E., Longoria, J., 2000, Magnetostratigraphy of mid-Cretaceous limestones from the Sierra Madre of northeastern Mexico: Geophysical Journal Internacional, $143,219-229$.

De Cserna, Z., 1970, Mesozoic sedimentation, magmatic activity, and deformation in northern Mexico, en Seewald, K., Sundeen, D. (eds.), The geologic framework of the Chihuahua tectonic belt: Midland, Texas, E.U.A., West Texas Geological Society, 99-117.

De Cserna, Z., 1976, Mexico geotectonics and mineral deposits: New Mexico Geological Society, Special Publication, 6, 18-25.

Eguiluz de Antuñano, S., 2001, Geologic Evolution and Gas Resources of the Sabinas Basin in Northeastern México, en Bartolini, C., Buffler, R.T., Cantú-Chapa, A. (eds.), The western Gulf of México Basin: Tectonics, sedimentary basins, and petroleum systems: American Association of Petroleum Geologists Memoir, 75, 241-270.

Fisher, R.A., 1953, Dispersion on a sphere: Proceedings of the Royal Society of London, A 217, 295-305.

Goldhammer, R.K., 1999, Mesozoic sequence stratigraphy and paleogeographic evolution of northeast of Mexico, en Bartolini, C., Wilson, J.L., Lawton, T.F. (eds.), Mesozoic Sedimentary and Tectonic History of North-Central Mexico: Boulder, Colorado, E.U.A., Geological Society of North America Special Paper, 340 , $1-58$.

González-Naranjo, G.A., 2006, Análisis estructural y estudio paleomagnético en el área Potrero El Colorado, porción occidental de la Sierra La Fragua, Coahuila, México: Linares, Nuevo León, Universidad Autónoma de Nuevo León, Tesis de Maestría, 221 p.

González-Naranjo, G.A., Molina-Garza, R.S., Chávez-Cabello, G., 2008, Paleomagnetic study of Jurassic and Cretaceous rocks north of San Marcos fault, central Coahuila, México: Geofísica Internacional, 47, 41-55.

Gradstein, F.M., Ogg, J.G., Smith, A.G., (eds.), 2005, A geologic time scale 2004: Cambridge, Reino Unido, Cambridge University Press, 610 p.

Gray, G.G., Lawton, T.F., Murphy, J.J., 2008, Looking for the MojaveSonora megashear in northeastern Mexico, en Moore, G. (ed.), Geological Society of America Field Guide, 14, 1-26.

Imlay, R.W., 1940, Neocomian faunas of Northern Mexico: Geological Society of America Bulletin, 51, 117-190.
Jones, N.W., McKee, J.W., Marquez, D.B., Tovar, J., Long, L.E., Laudon, T.S., 1984, The Mesozoic La Mula Island, Coahuila, México: Geological Society of America Bulletin, 95, 1226-1241.

Kirschvink, J.L., 1980, The least-square line and plane and the analysis of paleomagnetic data: Geophysical Journal of the Royal Astronomical Society, 62, 699-718.

Larson, E.E., Walker, T.R., 1982, A rock magnetic study of the lower massive sandstone, Moenkopi Formation (Triassic), Gray Mountain area, Arizona: Journal of Geophysical Research, 87, B6, 4819-4836.

Lehmann, C., Osleger, D.A., Montanez, I.P., Sliter, W., Arnaud-Vanneau, A., Banner, J., 1999, Evolution of Cupido and Coahuila carbonate platforms, Early Cretaceous, northeastern Mexico: Geological Society of America Bulletin, 111, 1010-1029.

López-Ramos, E., 1981, Paleogeografía y tectónica del Mesozoico en México: Universidad Nacional Autónoma de México, Revista del Instituto de Geología, 5, 158-177.

McElhinny, W., McFadden, P.L., 2000, Paleomagnetism: Continents and Oceans: San Diego, California, E.U.A., Academic Press, 386 p

McFadden, P.L., McElhinny, M.W., 1988, The combined analysis of remagnetization circles and direct observations in paleomagnetism: Earth and Planetary Science Letters, 87, 161-172.

McKee, J.W., Jones, N.W. 1979, A large Mesozoic fault in Coahuila, Mexico: Geological Society of America Abstracts with Programs, $11,476$.

McKee, J.W., Jones, N.W., Anderson, T.H., 1988, Las Delicias basin: A record of late Paleozoic arc volcanism in northeastern Mexico: Geology, 16, 37-40.

McKee, J.W., Jones, N.W., Long, L.E., 1984, History of recurrent activity along a major fault in northeastern Mexico: Geology, 12, 103-107.

McKee, J.W., Jones, N.W., Long, L.E., 1990, Stratigraphy and provenance of strata along the San Marcos fault, central Coahuila, Mexico: Geological Society of America Bulletin, 102, 593-614.

Murray, G.E., 1959, Introduction and regional geologic summary of fieldtrip area, southeastern Coahuila and western Nuevo Leon: South Texas Geological Society Field Trip Guidebook, A1-A4.

Opdyke, N.D., Channell, J.T., 1996, Magnetic Stratigraphy: San Diego, California, E.U.A., Academic Press, 346 p.

Padilla y Sánchez, R.J., 1982, Geologic evolution of the Sierra Madre Oriental between Linares, Concepción del Oro, Saltillo and Monterrey, Mexico: Austin, Texas, E.U.A., University of Texas, Tesis doctoral, $217 \mathrm{p}$.

Salvador, A., 1987, Late Triassic-Jurassic paleogeography and origin of Gulf of Mexico Basin: American Association of Petroleum Geologists Bulletin, 71, 419-451.

Wilson, J. L., 1990, Basement structural controls on Mesozoic carbonate facies in Northeastern México-a review, en Contribuciones al Cretácico de México y América Central: Actas de la Facultad de Ciencias de la Tierra - Universidad Autónoma de Nuevo León, 4, $5-45$.

Wilson, J.L., Pialli, G., 1977, A Lower Cretaceous shelf margin in northern Mexico, en Bebbout D.G., Loucks R.G. (eds.), Cretaceous carbonates of Texas and Mexico: University of Texas Bureau of Economic Geology Report of Investigations, 89, 302-323.

Zijderveld, J.D.A., 1967, A.C. demagnetization of rocks: analysis of results, en Collinson, D.W., Creer, K.M., Runcorn, S.K. (eds.), Methods in Paleomagnetism: Amsterdam, Holanda, Elsevier, 251-286.

Manuscrito recibido: Marzo 3, 2009.

Manuscrito corregido recibido: Septiembre 1, 2009.

Manuscrito aceptado: Noviembre 4, 2009. 